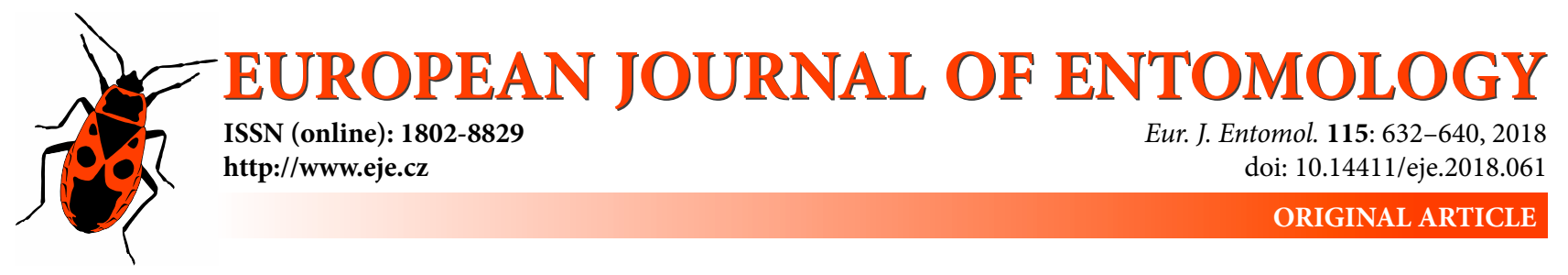

\title{
Detection of telomeric sequences and ribosomal RNA genes in holokinetic chromosomes of five jumping plant-lice species: First data on the superfamily Psylloidea (Hemiptera: Sternorrhyncha)
}

\author{
Anna MARYAŃSKA-NADAChOWSKA¹, Valentina G. KUZNETSOVA², Natalia V. GOLUB ${ }^{2}$ and Boris A. ANOKHIN \\ ${ }^{1}$ Institute of Systematics and Evolution of Animals, Polish Academy of Sciences, Sławkowska 17, 31-016 Kraków, Poland; \\ e-mail: maryanska@isez.pan.krakow.pl \\ ${ }^{2}$ Department of Karyosystematics, Zoological Institute, Russian Academy of Sciences, Universitetskaya nab. 1, \\ 199034 St. Petersburg, Russia; e-mails: valentina_kuznetsova@yahoo.com, psocid@zin.ru, cnidaria@zin.ru
}

Key words. Hemiptera, Sternorrhyncha, Psylloidea, Psyllidae, Aphalaridae, telomeric repeats, 18S rDNA, FISH, NOR

Abstract. Fluorescence in situ hybridization (FISH) is a technique used to determine the chromosomal position of DNA and RNA probes. The present study contributes to knowledge on jumping plant-lice genomes by using FISH with $18 \mathrm{~S}$ rDNA and telomeric $(\text { TTAGG })_{n}$ probes on meiotic chromosomes of Psylla alni $(2 n=24+X)$, Cacopsylla mali $\left(2 n=22+\right.$ neo- $X_{Y}$ and $\left.20+n e o-X_{1} X_{2} Y\right)$, C. sorbi $(2 n=20+$ neo-XY), Baeopelma foersteri $(2 n=14+X)$, and Rhinocola aceris $(2 n=10+X)$. This is the first study that has used FISH on the hemipteran superfamily Psylloidea. We found that the chromosomes of all studied species contain the insecttype telomere motif, (TTAGG). In C. mali and C. sorbi, the neo-sex chromosomes originating from autosome-sex chromosome fusions showed no interstitially located clusters of TTAGG repeats, suggesting their loss or inactivation. Similarly, no interstitial (TTAGG) ${ }_{n}$ clusters were detected in an extremely large autosome pair of $B$. foersteri that most likely originated from a fusion of at least five ancestral chromosome pairs. Clusters of $18 \mathrm{~S}$ rDNA were detected on the fused and second largest autosome pairs of $B$. foersteri and on one of the large autosome pairs of the remaining species. In $C$. mali and $B$. foersteri, the rDNA clusters were shown to coincide with the NORs as detected by the AgNOR method. Finally, we speculate, based on the obtained FISH markers, on the mechanisms of karyotype evolution of psylloid species differing in chromosome numbers and sex chromosome systems.

\section{INTRODUCTION}

The Psylloidea, or jumping plant-lice (Sternorrhyncha), are small sap-sucking hemipteran insects distributed worldwide. They feed on the phloem of vascular plants, with most species developing exclusively on dicotyledonous plants. Most psyllid species are oligophages restricted to one or a few closely related host plants, particularly at the larval stage. Several species are known to be harmful to their host plants; they can act as vectors for various plant diseases, as economically important pests in agriculture and forestry, and as potential biocontrol agents for some invasive plants. Approximately 4000 psyllid species have been described from across every biogeographic region of the world, most of which from tropical and subtropical regions (Burckhardt, 1987; Burckhardt \& Ouvrard, 2012; Burckhardt et al., 2014; Percy et al., 2018). The most recent classification of the Psylloidea (Burckhardt \& Ouvrard, 2012) recognizes 8 families: Aphalaridae, Carsidaridae, Calophyidae, Homotomidae, Liviidae, Phacopteronidae, Psyllidae, and Triozidae. Until now, approximately 175 psylloid species belonging to 55 genera of all families, except for the small family Phacopteronidae, have been analyzed cytogenetically (MaryańskaNadachowska \& Głowacka, 2005; Labina et al., 2007; Nokkala et al., 2008, 2013, 2017; for other references see Maryańska-Nadachowska, 2002). Jumping plant-lice are characterized by fairly uniform karyotypes with regard to both chromosome number, which is predominantly $25 / 26$ (male/female), and sex chromosome system, which is of an $\mathrm{X}(0) / \mathrm{XX}$ (male/female) type in more than $95 \%$ of species. Thus, the modal male karyotype of Psylloidea is $2 \mathrm{n}$ $=24+\mathrm{X}$, with the latter being regarded as ancestral for this superfamily (Maryańska-Nadachowska, 2002). All other karyotypes are attributable to derived conditions, and the variability seen among chromosome numbers and sex chromosome systems suggests that the deviated karyotypes have evolved independently in different lineages. With the exception of $2 \mathrm{n}=26+\mathrm{XY}$ found in the genera Pachypsylla Riley, 1883 (Aphalaridae) and Pauropsylla Rübsaamen, 1899 (Triozidae) (Walton, 1960; Maryańska-Nadachowska \& Głowacka, 2005), the number of autosomes in these karyotypes is lower than the modal number and ranges from 
6 to 20 in diploid complements. Several species display an $\mathrm{XY}$ sex chromosome system that is usually considered to be derived from the $\mathrm{X}(0)$ system by a fusion of the $\mathrm{X}$ chromosome with an autosome, whose homologue then became the Y chromosome (Grozeva \& Maryańska-Nadachowska, 1995; Maryańska-Nadachowska, 2002). At least two of the species, Cacopsylla mali (Schmidberger, 1836) and C. sorbi (Linnaeus, 1767), show sex chromosome polymorphism within populations, with males having either a neo-XY or neo- $X_{1} X_{2} Y$ system in the former species and either an $\mathrm{X}(0)$ or neo-XY system in the latter (Suomalainen \& Halkka, 1963; Grozeva \& Maryańska-Nadachowska, 1995; Maryańska-Nadachowska \& Grozeva, 2001). The chromosomes of jumping plant-lice are holokinetic (Suomalainen \& Halkka, 1963): these chromosomes lack physical landmarks such as primary constrictions (the centromeres) and therefore possess very few differentiating features, which greatly confines comparative cytogenetic approaches to this group. In recent years, different chromosome banding techniques (DNA-binding fluorochromes, C- and AgNOR-bandings) and especially fluorescence in situ hybridization (FISH) have made it possible to reveal chromosome-specific DNA markers for Hemiptera insects (reviewed in Mola \& Papeschi, 2006; Papeschi \& Bressa, 2006; Kuznetsova et al., 2012, 2015a, b; Kuznetsova \& Aguin-Pombo, 2015; Grozeva et al., 2015). In the jumping plant-lice species studied thus far, C-banding has not been able to induce clear or regular patterns of longitudinal differentiation along the chromosomes, while the number and location of the nucleolus organizer regions (NORs) seem to be highly uniform: the NORs are always present on one of the medium-sized pairs of autosomes in a sub-terminal position (reviewed in Maryańska-Nadachowska, 2002; see also: Kuznetsova et al., 2003; Labina et al., 2007). As such, the difficulty in acquiring useful results seems to have limited interest in these approaches. FISH, on the other hand, has never been used in cytogenetic investigations of Psylloidea.

The present paper provides the first cytogenetic knowledge on holokinetic chromosomes of Psylloidea studied with FISH technology. The aims were (1) to ascertain whether jumping plant-lice display the insect-type telomere motif, (TTAGG), (2) to analyze the chromosomal distribution of $18 \mathrm{~S}$ rDNA loci in five species belonging to four genera of two families, the Psyllidae and Aphalaridae, and (3) to speculate on the evolutionary trends and mechanisms of karyotype evolution of jumping plant-lice species with different chromosome numbers and sex chromosome systems, based on FISH markers.

\section{MATERIAL AND METHODS}

\section{Taxon sampling and fixation}

Adult males of five species were used in this study: Psylla alni (Linnaeus, 1758), Cacopsylla mali (Schmidberger, 1836), C. sorbi (Linnaeus,1767), Baeopelma foersteri Flor, 1861, and Rhinocola aceris (Linnaeus, 1758). All specimens were collected in May and June in 2015-2017 in Poland (Table 1) and identified by the senior author (AMN). Males were fixed in the field in a freshly mixed ethanol and glacial acetic acid mixture $(3: 1)$ and refrigerated in the laboratory at $-20^{\circ} \mathrm{C}$. The testes were extracted from the abdomen, placed on a slide in a drop of $45 \%$ acetic acid and squashed. The coverslips were removed with a razor blade after freezing with dry ice, and the slides then dehydrated in fresh fixative $(3: 1)$ and air dried.

\section{Fluorescence in situ hybridization (FISH)}

Probes for $18 \mathrm{~S}$ rDNA and (TTAGG) ${ }_{n}$ were prepared and FISH was performed according to Grozeva et al. (2011) with a few modifications, as described in our recent publications dealing with other hemipteran lineages, Auchenorrhyncha (Maryańska Nadachowska et al., 2013), Heteroptera (Kuznetsova et al., 2012; Angus et al., 2017) and Coleorrhyncha (Kuznetsova et al., $2015 b)$. In brief, the $18 \mathrm{~S}$ rDNA probe was amplified by PCR and labelled with biotin-11-dUTP (Fermentas, Vilnius, Lithuania) using genomic DNA of the true bug Pyrrhocoris apterus (Linneus, 1758): an initial denaturation period of $3 \mathrm{~min}$ at $94^{\circ} \mathrm{C}$ was followed by 33 cycles of $30 \mathrm{~s}$ at $94^{\circ} \mathrm{C}$, annealing for $30 \mathrm{~s}$ at $50^{\circ} \mathrm{C}$ and extension for $1.5 \mathrm{~min}$ at $72^{\circ} \mathrm{C}$, with a final extension step of $3 \mathrm{~min}$ at $72^{\circ} \mathrm{C}$. The telomere probe (TTAGG) was amplified by PCR and labeled with rhodamine-5-dUTP (GeneCraft, Köln, Germany): an initial denaturation period of $3 \mathrm{~min}$ at $94^{\circ} \mathrm{C}$ was followed by 30 cycles of $45 \mathrm{~s}$ at $94^{\circ} \mathrm{C}$, annealing for $30 \mathrm{~s}$ at $50^{\circ} \mathrm{C}$ and extension for $50 \mathrm{~s}$ at $72^{\circ} \mathrm{C}$, with a final extension step of 3 $\min$ at $72^{\circ} \mathrm{C}$. For primers, see Grozeva et al. (2011). The chromosome preparations were treated with $100 \mu \mathrm{g} / \mathrm{ml}$ RNase A and 5 $\mathrm{mg} / \mathrm{ml}$ pepsin solution to remove excess RNA and proteins. Chromosomes were denatured in the hybridization mixture containing labelled 18S rDNA and (TTAGG), probes with an addition of salmon sperm blocking reagent and then hybridized for $42 \mathrm{~h}$ at $37^{\circ} \mathrm{C} .18 \mathrm{~S}$ rDNA probes were detected with NeutrAvidin-Fluorescein conjugate (Invitrogen, Karlsbad, CA, USA). The chromosomes were mounted in an antifade medium (ProLong Gold antifade reagent with DAPI, Invitrogen) and covered with a glass coverslip.

The number of males involved in the study ranged from 4 to 7 and the number of prophase/metaphase plates examined ranged from 20 to 34 in different species (Table 1).

\section{AgNOR-banding}

Only two species, C. mali and B. foersteri, were studied by this method. The chromosome slides were incubated with $50 \%$ $\mathrm{AgNO}_{3}$ and $1 \%$ gelatin developer at $60^{\circ} \mathrm{C}$ for $7-8 \mathrm{~min}$, rinsed in distilled water, air-dried, and mounted in Entellan (Merck, Darmstadt, Germany).

Table 1. Material studied.

\begin{tabular}{|c|c|c|c|}
\hline Species & Collection localities & Host plant & Number of males/nuclei \\
\hline Psylla alni (Linnaeus, 1758) & Poland, Niepołomice, $50^{\circ} 03^{\prime} 25^{\prime \prime} \mathrm{N} / 20^{\circ} 21^{\prime} 66^{\prime \prime} \mathrm{E}$ & Alnus incana & $4 / 24$ \\
\hline Cacopsylla mali (Schmidberger, 1836) & Poland, Kraków, 5008’33"N/1991’67"E & Malus sp. & $6 / 22$ \\
\hline Cacopsylla sorbi (Linnaeus,1767) & Poland, Kraków, 5008’33"N/1991’67"E & Sorbus aucuparia & $5 / 20$ \\
\hline Baeopelma foersteri Flor, 1861 & Poland, Nowa Biała, $49^{\circ} 45^{\prime} 00^{\prime \prime} \mathrm{N} / 20^{\circ} 15^{\prime} 00^{\prime \prime} \mathrm{E}$ & Alnus glutinosa & $7 / 27$ \\
\hline Rhinocola aceris (Linnaeus, 1758) & Poland, Kraków, 508'33"N/1991'67"E & Acer platanoides & $5 / 34$ \\
\hline
\end{tabular}




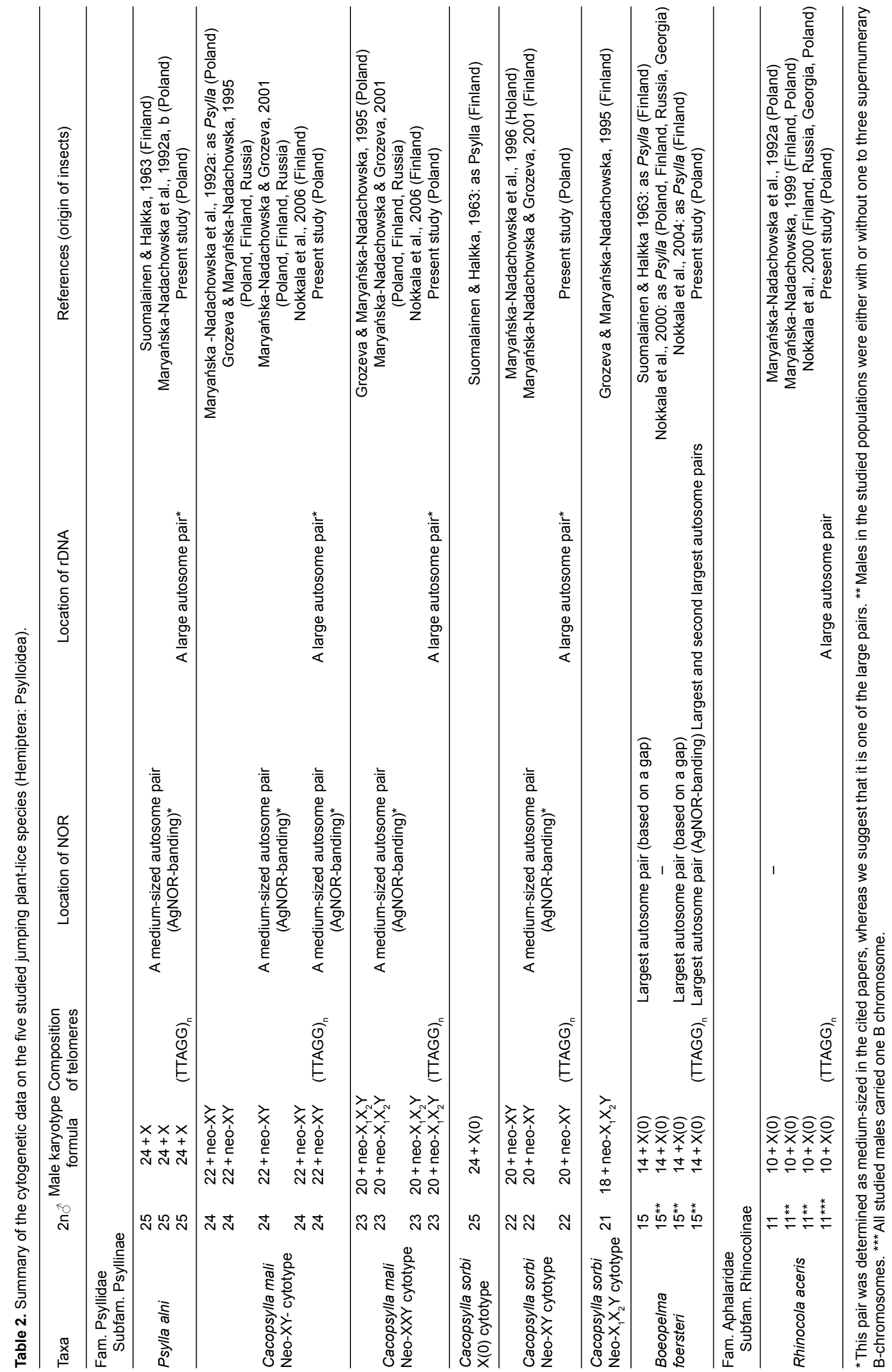



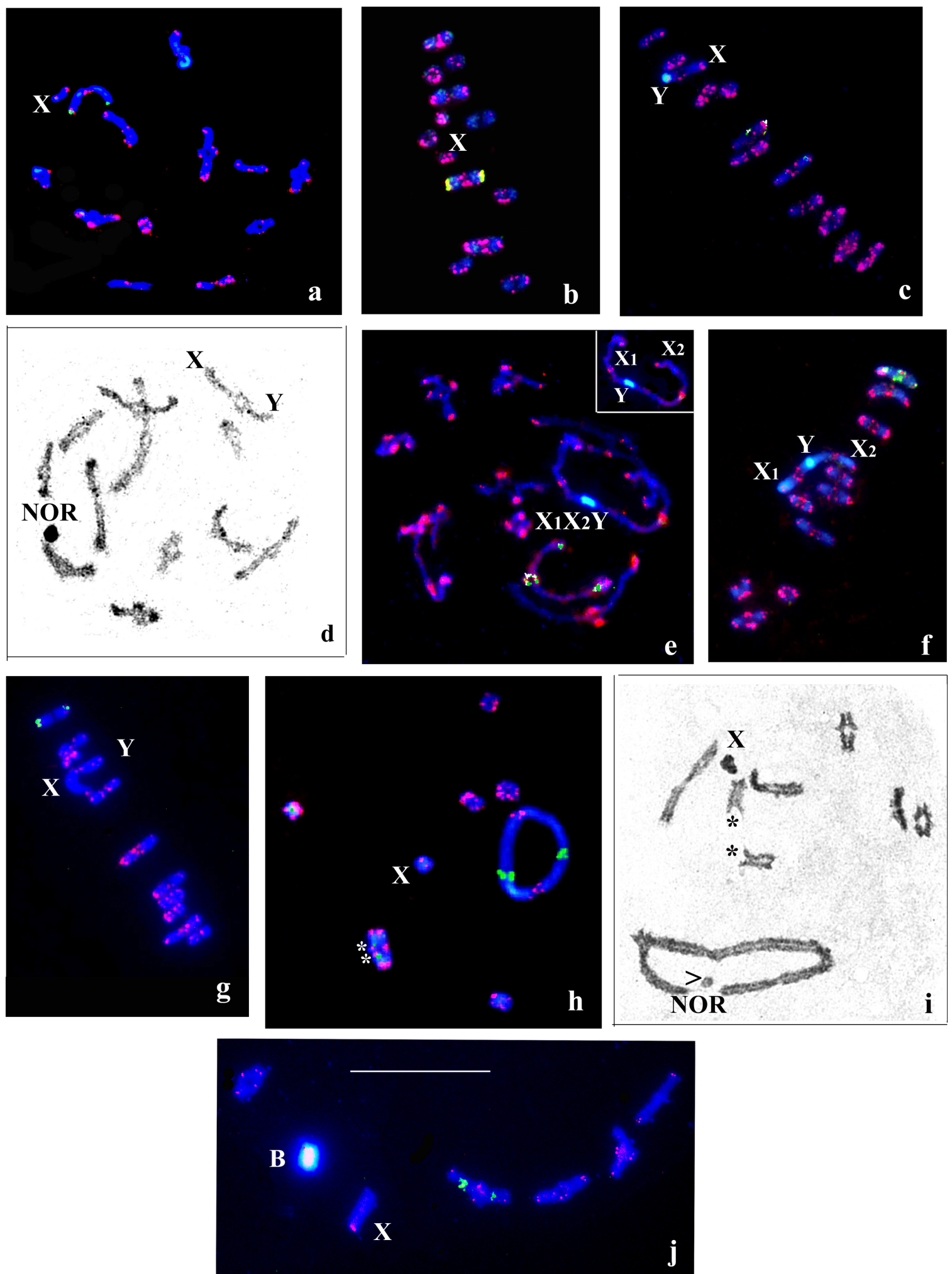

Fig. 1. Examples of FISH with rDNA (green signals) and (TTAGG) telomeric (red signals) probes (a-c, e-h, j) and those of AgNOR-banding (d, i) in male meiotic chromosomes of five jumping plant-lice species (Psylloidea). Psylla alni, $\mathrm{n}=12+\mathrm{X}(\mathrm{a}, \mathrm{b})$ : diakinesis/metaphase I (a) and metaphase l/anaphase I (b). rDNA sites are located sub-terminally/terminally on both homologues of a large bivalent; Cacopsylla mali, the neo-XY cytotype, $2 \mathrm{n}=11+\mathrm{neo-} \mathrm{XY}(\mathrm{c}, \mathrm{d})$ : metaphase I/anaphase I, rDNA sites are located sub-terminally/terminally on both homologues of a large bivalent, one homologue shows rDNA-site at the chiasmate region of the bivalent while the other homologue shows it on the opposite region of the bivalent, DAPI-positive region is present on the neo-Y (c), diplotene/diakinesis, NOR is located on a large bivalent (d); C. mali, the $X_{1} X_{2} Y$ cytotype, $n=10+$ neo- $X_{1} X_{2} Y(e, f)$ : diplotene/diakinesis, rDNA-sites are located on both ends of every homologue of a large bivalent, DAPI-positive region is seen at least on $Y$ chromosome (e, framed is neo- $X, X_{2} Y$ trivalent), metaphase I, rDNA-sites are located on both ends of every homologue of a large bivalent, DAPI-positive regions are seen on $X_{1}$ and $Y$ chromosomes (f); Cacopsylla sorbi, the $X Y$ cytotype, $n=10+$ neo-XY: metaphase I/anaphase I, rDNA-sites are located sub-terminally/terminally on both homologues of a large bivalent (g); Baeopelma foersteri, $\mathrm{n}=7+\mathrm{X}(\mathrm{h}$, i): diakinesis/metaphase I, rDNA sites are located interstitially on both homologues of the largest bivalent, the second largest bivalent displays sub-terminal/terminal rDNA sites on both homologues as well (asterisks) (h), diakinesis, NOR is located on the largest bivalent ( $>$ indicates a nucleolus remnant), the homologues of the second largest bivalent are present as univalents (asterisks) being most probably connected by thin threads with NOR (i); Rhinocola aceris, $n=5+X+B$ : diakinesis/metaphase I, rDNA sites are located interstitially on both homologues of a large bivalent, B chromosome is DAPI-positive (j). Bar $=10 \mu \mathrm{m}$. 


\section{Microscopy and imaging}

$\mathrm{AgNO}_{3}$-banded slides were analyzed using an Olympus BX 51 light microscope with an Olympus C-35 AD-4 camera. FISH images were taken using a Leica DM $6000 \mathrm{~B}$ microscope with a $100 \times$ objective, Leica DFC 345 FX camera, and Leica Application Suite 3.7 software with an Image Overlay module (Leica Microsystems, Wetzlar, Germany). The filter sets applied were A, L5 and N21 (Leica Microsystems).

\section{RESULTS}

FISH analyses were carried out on a total of 27 males of P. alni, C. mali (two cytotypes), C. sorbi, B. foersteri, and $R$. aceris, which differed from each other in chromosome number and sex chromosome system. The first four species belong to the largest family Psyllidae, while the lattermost species to the medium-sized family Aphalaridae. In two species, $C$. mali and B. foersteri, the number and location of NORs were additionally examined using the AgNOR method. The basic data obtained in this study, together with chromosome data available in the literature for each of the above species, are summarized in Table 2. Representative images of meiotic karyotypes subjected to AgNOR-banding and FISH with the $18 \mathrm{~S}$ rDNA and (TTAGG) ${ }_{n}$ probes are shown in Fig. 1a-j.

\section{Psylla alni}

$2 \mathrm{n}=24+X($ Fig. 1a, b)

Four males and 24 prophase/metaphase I cells were studied, all showing 12 autosomal bivalents and an X-univalent (Fig. 1a, b: $\mathrm{n}=12+\mathrm{X}$ ). Approximately three larger bivalents could be distinguished, with the $\mathrm{X}$ being one of the small chromosomes of the set. Each bivalent formed one sub-terminal/terminal chiasma. FISH revealed TTAGG telomeric repeats in all plates, the signals were single, double or sometimes numerous on the chromosomes. Moreover, they could be absent on one of the homologues or on one of the ends of a homologue. 18S rDNA-FISH sites were located sub-terminally/terminally on a large bivalent.

\section{Cacopsylla mali}

$2 \mathrm{n}=22+$ neo-XY (Fig. 1c, d) $; 2 \mathrm{n}=20+$ neo-X1X2Y (Fig. 1e, f) (see explanation in the discussion)

Six males, 18 prophase/metaphase I and 4 metaphase II nuclei were studied. Although all males were collected at the same time from the same Malus sp. tree, four of them showed 11 autosomal bivalents and a heteromorphic sex chromosome bivalent (Fig. 1c, d: $n=11+\mathrm{XY}$ ), while the two remaining males had one less autosomal bivalent and a trivalent consisting of three sex chromosomes (Fig. 1e, $\mathrm{f}: \mathrm{n}=10+\mathrm{X}_{1} \mathrm{X}_{2} \mathrm{Y}$ ). In the former karyotype (the XY cytotype), the sex chromosome bivalent is one of the largest elements of the set, whereas in the latter (the $\mathrm{X}_{1} \mathrm{X}_{2} \mathrm{Y}$ cytotype), the sex trivalent is notably larger than the sex bivalent in the former. Each autosomal bivalent formed one sub-terminal/terminal chiasma. In both cytotypes, FISH revealed TTAGG repeats on all autosomal bivalents and on the neo-sex chromosomes. Furthermore, the neo-Y chromosome of the neo-XY cytotypes and both the neo- $\mathrm{Y}$ and neo- $\mathrm{X}_{1}$ chromosomes of the $\mathrm{X}_{1} \mathrm{X}_{2} \mathrm{Y}$ cytotype were found to carry large DAPI-positive blocks of heterochromatin (Fig. 1c, e, f). 18S rDNA-FISH revealed the presence of rDNA on one of the large bivalents in both cytotypes. In the XY cytotype, the rDNA signals were present on one end of every homologue, whereas in the $\mathrm{X}_{1} \mathrm{X}_{2} \mathrm{Y}$ cytotype both ends of every homologue showed signals of rDNA. Certain bivalents also appeared to carry additional hybridization signals of rRNA genes, which were generally very small and inconstant (not shown). In the XY cytotype, one of the large bivalents, most likely that which showed prominent rDNA-FISH signals, carried NORs as detected by the AgNOR technique (Fig. 1d).

\section{Cacopsylla sorbi}

$2 \mathrm{n}=20+$ neo-XY (Fig. 1g) (see explanation in the discussion)

Five males, 12 prophase/metaphase I and 8 metaphase II nuclei were studied. Males showed 10 autosomal bivalents and a heteromorphic sex chromosome bivalent in the first meiotic division (Fig. 1g: $n=10+X Y$ ). Among the autosomal bivalents, at least 6 larger and 4 smaller ones could be distinguished, with the XY pair being the largest element of the karyotype. Each autosomal bivalent formed one sub-terminal/terminal chiasma. FISH revealed the presence of TTAGG telomeric repeats on all chromosomes, however, some bivalents lacked visible signals on separate homologues. While a clear $18 \mathrm{~S}$ rDNA-FISH signals were located sub-terminally/terminally on one of the large bivalents.

\section{Baeopelma foersteri}

\section{$2 \mathrm{n}=14+X($ Fig. 4h, i)}

Seven males, 24 prophase/metaphase I and 3 metaphase II nuclei were studied. Males showed 7 bivalents and a univalent $\mathrm{X}$ chromosome in the first meiotic division (Fig. 1h, $\mathrm{i}: \mathrm{n}=7+\mathrm{X}$ ). One of the bivalents was several times larger than the second largest bivalent of the karyotype. The $\mathrm{X}$ chromosome was one of the smallest elements of the set. Each bivalent formed a single sub-terminal/terminal chiasma and only the largest bivalent had two sub-terminal/ terminal chiasmata in every nucleus. FISH revealed the presence of TTAGG telomeric repeats on all chromosomes. 18S FISH signals were located interstitially on each homologue of the largest bivalent, with the second largest bivalent also showing rDNA sites. After silver staining, the position of NOR appeared to coincide with that of the rDNA-FISH signals on the largest bivalent (Fig. 1i). The homologues of the second largest bivalent were present as univalents (Fig. 1i), probably being connected by thin threads to another NOR.

\section{Rhinocola aceris}

\section{$2 \mathrm{n}=10+X($ Fig. $1 \mathrm{j})$}

Five males, 30 prophase/metaphase I and 4 metaphase II nuclei were studied. In all males, the karyotype consisted of 5 bivalents and a univalent $\mathrm{X}$ chromosome as well as a rather large and heterochromatic $\mathrm{B}$ chromosome in the first meiotic division (Fig. 1j: $n=5+B+X$ ). Each bivalent was observed to form one sub-terminal/terminal chiasma. The 
$\mathrm{X}$ chromosome is large and most probably exceeds in size the majority of chromosomes in the karyotype. FISH revealed the presence of TTAGG telomeric repeats on chromosomes; however some, including the $\mathrm{X}$, displayed no visible signals on one or both ends. No evident TTAGG signals were present on the B chromosome. 18S rDNAFISH signals were located interstitially on one of the largest bivalents.

\section{DISCUSSION}

\section{Standard karyotypes}

The standard karyotypes of species examined in the present study have previously been reported (see Table 2); the data obtained herein coincide with the previous descriptions. In the five species studied we found six different karyotypes which represent almost all of the basic variants currently known in jumping plant-lice species. The male karyotype $2 \mathrm{n}=24+\mathrm{X}$ of Psylla alni is known to be a conserved pattern found in most species and is considered to be evolutionarily ancestral for Psylloidea (MaryańskaNadachowska, 2002). Cacopsylla mali and C. sorbi are unique within the superfamily in having derived sex chromosome systems originating from one or several $\mathrm{X}$ autosome fusions (Grozeva \& Maryańska-Nadachowska, 1995). For Cacopsylla sorbi, previous studies described two male karyotypes: $2 \mathrm{n}=24+\mathrm{X}$ in two males examined in a population from Finland (Suomolainen \& Halkka, 1963 ) and $2 \mathrm{n}=20+$ neo-XY in several European populations, including that from Finland (Grozeva \& MaryańskaNadachowska, 1995; Maryańska-Nadachowska et al., 1996; Maryańska-Nadachowska \& Grozeva, 2001). The latter chromosome form (XY cytotype) studied in the present work could have originated from $2 n=24+X$ through at least two fusions, one of them between two pairs of ancestral autosomes resulting in $22+\mathrm{X}$ (not yet found), and another between one of the fusion chromosomes and the X. In Cacopsylla mali, a fusion first occurred between an autosomal pair and the $\mathrm{X}$ in the progenitor karyotype $2 \mathrm{n}=24$ $+\mathrm{X}$, resulting in $2 \mathrm{n}=22+$ neo-XY. A further fusion then occurred between the neo-XY and another autosomal pair resulting in $2 n=20+$ neo- $X_{1} X_{2} Y$ (Grozeva \& MaryańskaNadachowska, 1995). Remarkably, males with different karyotypes (neo-XY and neo- $\mathrm{X}_{1} \mathrm{X}_{2} \mathrm{Y}$ cytotypes) were found to coexist within different geographically distant populations while being morphologically indistinguishable from each other (Grozeva \& Maryańska-Nadachowska, 1995; Maryańska-Nadachowska \& Grozeva, 2001; Nokkala et al., 2004; present study). One may speculate that males displaying neo- $\mathrm{XY}$ and neo- $\mathrm{X}_{1} \mathrm{X}_{2} \mathrm{Y}$ systems belong to different coexisting sex chromosome races or even cryptic species; however, such a suggestion is highly improbable. These males are most likely conspecific. A reason for the neo- $\mathrm{X}_{1} \mathrm{X}_{2} \mathrm{Y}$ system to be present in a polymorphic state (together with the neo-XY system) in different geographically distant populations of Cacopsylla mali, has been attributed to the high frequency of unbalanced gametes produced during meiosis in $\mathrm{X}_{1} \mathrm{X}_{2} \mathrm{Y}$ individuals, thus resulting in the neo- $X_{1} X_{2} Y$ system being unable to become fixed in a population (Nokkala et al., 2004).

Baeopelma foersteri and Rhinocola aceris had lower than modal chromosome numbers, namely $2 \mathrm{n}=14+\mathrm{X}$ and $2 \mathrm{n}=10+\mathrm{X}$ (male), respectively. In addition, some $R$. aceris populations (including the one studied here) are known to have B chromosomes (reviewed in MaryańskaNadachowska, 1999). Previous studies of B chromosomes in $R$. aceris have provided interesting insights into their evolutionary importance: incorporation of B chromosome in achiasmate segregation mechanisms with the $\mathrm{X}$ chromosome in different populations shows consecutive stages of its conversion into a Y chromosome (Nokkala et al., 2000; Nokkala \& Nokkala, 2004). In B. foersteri, the presence of an extremely large chromosome pair equivalent in size to some 5 or 6 standard chromosomes (Suomalainen \& Halkka, 1963) is certainly an evidence of the chromosome fusions which have occurred in its karyotype evolution. Taking $2 \mathrm{n}=25$ to be the ancestral karyotype of Psylloidea (Maryańska-Nadachowska, 2002), a total of 5 fusions between autosomal pairs are required to produce the $B$. foersteri karyotype $2 \mathrm{n}=15$ from a species with 12 pairs of bivalents.

\section{Characterization of karyotypes using FISH with telomeric and $18 S$ rDNA probes}

In recent years, FISH has been expanding our understanding of the genetic basis of karyotype evolution in insects with holokinetic chromosomes. Our study represents the first application of this method to the chromosomes of jumping plant-lice. Both the telomeric sequence (TTAGG) and ribosomal DNA (18S rDNA probe) were physically mapped in five species differing in chromosome numbers and sex chromosome systems. Telomeres of all studied species were observed to have the (TTAGG) motif. This tandemly repeated TG-rich pentanucleotide sequence is known to be generally characteristic (albeit not universal) of Insecta and is considered to be an ancestral DNA motif of the telomeres of insects and also all arthropods (Sahara et al., 1999; Frydrychová \& Marec, 2002; Frydrychová et al., 2004; Vítková et al., 2005). Taken together with our present data on Psylloidea, the (TTAGG) motif is currently known to be encountered in all major Hemiptera clades, including (in addition to Psylloidea) Coccoidea (Mohan et al., 2011), Aphidoidea (Bizzaro et al., 2000; Frydrychová et al., 2004; Monti et al., 2011; Manicardi et al., 2015), Aleyrodoidea (Frydrychová et al., 2004; Luan et al., 2018), Auchenorrhyncha (Frydrychová et al., 2004; Maryańska-Nadachowska et al., 2013, 2016; Golub et al., 2014; Kuznetsova et al., 2015a, c; Anjos et al., 2016), Coleorrhyncha (Kuznetsova et al., 2015b), and Heteroptera, in which at least all the more basal groups studied to date have this motif (Kuznetsova et al., 2012; Chirino et al., 2017; Angus et al., 2017). These findings strongly support the ancestral nature of the so-called insect telomeric motif (TTAGG) $_{n}$ in the monophyletic order Hemiptera.

In addition to the canonical DNA sequences located at the terminal ends of chromosomes, non-terminal TTAGG repeats, known as interstitial telomeric sequences (ITSs), 
have been observed in many eukaryotes, from yeasts to humans, and are often considered to be relicts of the tandem fusion of ancestral chromosomes during karyotype evolution (reviewed by Lin \& Yan, 2008). In insects, ITSs consisting of the (TTAGG) motif have so far been identified in only a few species. This includes those with holokinetic chromosomes such as the moth Orgyia antiqua (Linnaeus, 1758) (Rego \& Marec, 2003) and three species of the giant water bug genus Belostoma Latreille, 1807 (Chirino et al., 2017), all with reduced chromosome numbers resulting from autosome fusions. Considering that the telomeres are responsible for chromosome stability and integrity, it can be hypothesized that a prerequisite for the formation of end-to-end chromosome fusions should be either the inactivation or elimination of telomeres (Chirino et al., 2017 and references therein). In support of this, a study by Rego \& Marec (2003) found no ITSs on a fused chromosome of a different moth species, Ephestia kuehniella (Zeller, 1879), suggesting that the telomeres had probably been lost prior to the fusion of the two original chromosomes. Data obtained in our study also strengthen the aforementioned hypothesis. Both the neo-XY bivalents and neo- $\mathrm{X}_{1} \mathrm{X}_{2} \mathrm{Y}$ trivalents of Cacopsylla sorbi and C. mali displayed hybridization signals of the (TTAGG) ${ }_{n}$ probe, clearly demarcating the telomeric regions of chromosomes involved in the neosex chromosome systems. However these chromosomes showed no ITSs despite originating from several fusions between autosomes and sex-chromosomes (Grozeva \& Maryańska-Nadachowska, 1995). Similarly, no hybridization signals of the telomeric probe were revealed in the interstitial sites of the fused chromosome pair in $B$. foersteri that has a reduced chromosome number $(2 n=15)$. The absence of ITSs may indicate that the telomeric repeats were lost before the original chromosomes fused, as has been suggested for Ephestia kuehniella (Rego \& Marec, 2003), or that they were inactivated (for other possible explanations for the phenomenon, see Chirino et al., 2017). Moreover, it cannot be excluded that these sequences are in fact present on the fused chromosomes in such a low copy number as to be undetectable by FISH analyses.

The psyllid species examined in our study showed a considerable variation in the number and intensity of the TTAGG signals on almost all chromosomes, these differences also being observed between homologues of a bivalent. Such a variation has been recorded for the giant water bug genus Belostoma (Heteroptera) presumably due to ascribed differences in the length of target TTAGG sequences and/or differences in hybridization efficiencies of the FISH experiments performed (Chirino et al., 2017).

In all species (with the exception of Baeopelma foersteri) the major rDNA clusters were revealed on a single pair of autosomes. In Psylla alni, Cacopsylla sorbi, and $C$. mali, the clusters were located sub-terminally/terminally on one of the large pairs of autosomes, and NORs, when studied by the AgNOR method, appeared to be similarly located on a large bivalent in these species (MaryańskaNadachowska et al., 1992a, b; Maryańska-Nadachowska \& Grozeva, 2001; present study). Unfortunately, with no additional markers it is difficult to speculate whether this pair is the same (homeologous) in different karyotypes and thus whether the species share a syntenic location of their rDNA arrays. It is noteworthy that in the neo-XY cytotype of $C$. mali small additional hybridization signals of rDNAFISH were present on different chromosomes, thus suggesting that the genome of this species contains an amount of RNA cistrons distributed along various chromosomes. Moreover, only a single NOR, as revealed by silver staining, was transcriptionally active during the preceding interphase (Goodpasture \& Bloom, 1975). In contrast to the above three species, both species with a reduced number of chromosomes, Rhinocola aceris and Baeopelma foersteri, were shown to have interstitially located rDNA clusters; in the former these were housed on one of the largest pairs of autosomes, and for the latter, on both the largest and the second largest pairs of autosomes. FISH results combined with those of silver staining appeared to indicate that it was a NOR-bearing pair of autosomes which had been involved in the fusion events during the evolution of B. foersteri.

In conclusion, the present study contributes to knowledge on Psylloidea genomes by reporting for the first time the distribution of rDNA clusters and (TTAGG) ${ }_{n}$ telomeric sequences in holokinetic chromosomes of five species with different karyotypes. Moreover, the data provide hitherto missing evidence that the insect type (TTAGG) ${ }_{n}$ telomeric motif is an ancestral trait for all Hemiptera. Future karyotype analyses using FISH should be undertaken on other jumping plant-lice species to gain a more comprehensive view of karyotype evolution of the superfamily Psylloidea.

ACKNOWLEDGEMENTS. Financial support for this study was provided to the Zoological Institute of the Russian Academy of Sciences by grant no. 14-14-00541 from the Russian Science Foundation. Per-article publication fee was paid by Institute of Systematics and Evolution of Animals PAN, Kraków, Poland. Authors AMN and VGK contributed equally to the study.

\section{REFERENCES}

Angus R.B., Jeangirard C., Stoianova D., Grozeva S. \& KuznetSOVA V.G. 2017: A chromosomal analysis of Nepa cinerea Linnaeus, 1758 and Ranatra linearis (Linneaus, 1758) (Heteroptera, Nepidae). - Comp. Cytogenet. 11: 641-657.

Anjos A., Rocha G.C., Paladini A., Mariguela T.C. \& CabralDE-MeLLo D.C. 2016: Karyotypes and repetitive DNA evolution in six species of the genus Mahanarva (Auchenorrhyncha: Cercopidae). - Cytogenet. Genome Res. 149: 321-327.

Bizzaro D., Mandrioli M., Zanotti M., Giusti M. \& Manicardi G.C. 2000: Chromosome analysis and molecular characterization of highly repeated DNAs in the aphid Acyrthosiphon pisum (Aphididae, Hemiptera). - Genetica 108: 197-202.

Burckhardt D. 1987: Jumping plant lice (Homoptera: Psylloidea) of the temperate neotropical region. Part 2: Psyllidae (subfamilies Diaphorininae, Acizziinae, Ciriacreminae and Psyllinae). - Zool. J. Linn. Soc. 90: 145-205.

BurCKHARDT D. \& OUVRARD D. 2012: A revised classification of the jumping plant-lice (Hemiptera: Psylloidea). — Zootaxa 3509: $1-34$.

Burckhardt D., Ouvrard D., Percy D. \& Queiroz D. 2014: Psyllid host-plants (Hemiptera: Psylloidae): resolving a semantic problem. — Fla Entomol. 97: 242-246. 
Chirino M.G., Dalíková M., Marec F. \& Bressa M. 2017: Chromosomal distribution of interstitial telomeric sequences as signs of evolution through chromosome fusion in six species of the giant water bugs (Hemiptera, Belostoma). - Ecol. Evol. 7: 5227-5235.

Frydrychová R. \& MAReC F. 2002: Repeated losses of TTAGG telomere repeats in evolution of beetles (Coleoptera). - Genetica 115: 179-187.

Frydrychová R., Grossmann P., Trubač P., VítKová M. \& Mared F. 2004: Phylogenetic distribution of TTAGG telomeric repeats in insects. - Genome 47: 163-178.

Golub N.V., Kuznetsova V.G. \& Rakitov R.A. 2014: First karyotype data on the family Myerslopiidae (Hemiptera, Auchenorrhyncha, Cicadomorpha). - Comp. Cytogenet. 8: 293-300.

Goodpasture C. \& Bloom S.E. 1975: Vizualization of nucleolar organizer regions in mammalian chromosomes using silver staining. - Chromosoma 53: 37-50.

Grozeva S.M. \& MaryańSKa-NadachowsKa A. 1995: Meiosis of two species of Cacopsylla with polymorphic sex chromosomes in males (Homoptera, Psyllidae). — Folia Biol. (Kraków) 43: 93-98.

Grozeva S., Kuznetsova V.G. \& Anokhin B.A. 2011: Karyotypes, male meiosis and comparative FISH mapping of $18 \mathrm{~S}$ ribosomal DNA and telomeric (TTAGG) ${ }_{n}$ repeat in eight species of true bugs (Hemiptera, Heteroptera). - Comp. Cytogenet. 5: 355-374.

Grozeva S., Anokhin B. \& Kuznetsova V.G. 2015: Bed bugs (Hemiptera). In Sharachov I. (ed.): Protocols for Cytogenetic Mapping of Arthropod Genomes. CRC press, Taylor \& Francis, Boca Raton, pp. 285-326.

Kuznetsova V. \& Aguin-Pombo D. 2015: Comparative cytogenetics of Auchenorrhyncha (Hemiptera, Homoptera): a review. ZooKeys 538: 63-93.

Kuznetsova V.G., Maryańska-Nadachowska A. \& Nokkala S. 2003: A new approach to the Auchenorrhyncha (Hemiptera, Insecta) cytogenetics: chromosomes of the meadow spittlebug Philaenus spumarius (L.) examined using various chromosome banding techniques. - Folia Biol. (Kraków) 51: 33-40.

Kuznetsova V.G., Grozeva S.M. \& Anokhin B.A. 2012: The first finding of (TTAGG) ${ }_{n}$ telomeric repeat in chromosomes of true bugs (Heteroptera, Belostomatidae). - Comp. Cytogenet. 6: 341-346.

Kuznetsova V.G., Maryańska-Nadachowska A. \& KaramysheVA T. 2015a: Spittlebugs (Hemiptera). In Sharakhov I. (ed.): Protocols for Cytogenetic Mapping of Arthropod Genomes. CRC Press, Taylor \& Francis Group, Boca Raton, pp. 351-380.

Kuznetsova V.G., Grozeva S.M., Hartung V. \& Anokhin B.V. 2015b: First evidence for (TTAGG) ${ }_{n}$ telomeric sequence and sex chromosome postreduction in Coleorrhyncha (Insecta, Hemiptera). - Comp. Cytogenet. 9: 523-532.

Kuznetsova V.G., Maryańska-Nadachowska A., Anokhin B.V. \& Aguin-Pombo D. 2015c: Evidence for TTAGG telomere repeats and rRNA gene clusters in leafhoppers of the genus Alebra (Hemiptera: Auchenorrhyncha: Cicadellidae). - Eur. J. Entomol. 112: 207-214.

Labina E.S., MaryańsKa-NadachowsKa A. \& Kuznetsova V.G. 2007: Meiotic karyotypes in males of nineteen species of Psylloidea (Hemiptera) in the families Psyllidae and Triozidae. Folia Biol. (Kraków) 55: 27-34.

LiN K.W. \& YAN J. 2008: Ending in the middle: Current knowledge of interstitial telomeric sequences. - Mutat. Res. 658: 95-110.

Luan J., Sun X., Fei Z. \& Douglas A.E. 2018: Maternal inheritance of a single somatic animal cell displayed by the bacteriocyte in the whitefly Bemisia tabaci. — Curr. Biol. 28: 459-465.
Manicardi G.C., Mandrioli M. \& Blackman R.L. 2015: The cytogenetic architecture of the aphid genome. - Biol. Rev. 90: $112-125$.

MARYAŃSKA-NADACHOWSKA A. 1999: B-chromosome polymorphism in Rhinocola aceris (Psylloidea, Homoptera). - Folia Biol. (Kraków) 47: 115-121.

MARYAŃSKA-NADACHOWSKA A. 2002: Review of karyotype variation in jumping plant-lice (Psylloidea, Sternorrhyncha, Hemiptera) and checklist of chromosome numbers. - Folia Biol. (Kraków) 50: 135-152.

MaryańSKa-NadachowsKa A. \& GŁowacka E. 2005: Meiotic karyotypes and structure of testes of nineteetn species of jumping-lice (Hemiptera, Psylloidea) from South Africa. — Folia Biol. (Kraków) 53: 143-148.

MaryańSKa-NadachowsKa A. \& GrozeVa S. 2001: Patterns of Cand $\mathrm{AgNO}_{3}$ bands in Cacopsylla mali (Schmidberger) and $C$. sorbi (Linnaeus) (Homoptera, Psyllidae) - species with polymorphic sex chromosome system. - Folia Biol. (Kraków) 49: $157-161$.

MaryańsKa-NadachowsKa A., KuZnetsova V.G. \& WarchałowSKA-Śliwa E. 1992a: Karyotypes of Psyllina (Homoptera). I. New data and check-list. - Folia Biol. (Kraków) 40: 15-25.

MARYAŃSKA-NADACHOWSKA A., WARChALOWSKA-ŚlIWA E. \& KuzNetsova V.G. 1992b: The NOR and nucleolus in the spermatogenesis of Psyla alni (L.) (Homoptera) analysed by silver staining. - Folia Biol. (Kraków) 40: 41-45.

Maryańska-Nadachowska A., Kuznetsova V.G., Yang C.T. \& WoudstRA I.H. 1996: New data on karyotypes and the number of testicular follicles in the psyllid families Aphalaridae, Psyllidae, Carsidaridae, and Triozidae (Homoptera, Psylloidea). Caryologia 49: 279-285.

MaryańsKa-NadachowsKa A., KuZnetsova V.G. \& KaramySHEVA T.V. 2013: Chromosomal location of rDNA clusters and TTAGG telomeric repeats in eight species of the spittlebug genus Philaenus (Hemiptera: Auchenorrhyncha: Aphrophoridae). - Eur. J. Entomol. 110: 411-418.

MaryańsKa-NadachowsKa A., Anokhin B.A., Gnezdilov V.M. \& Kuznetsova V.G. 2016: Karyotype staility in the family Issidae (Hemiptera, Auchenorrhyncha) revealed by chromosome techniques and FISH with telomeric (TTAGG) ${ }_{n}$ and $18 \mathrm{~S}$ rDNA probes. - Comp. Cytogenet. 10: 347-369.

Mohan K.N., Rani B.S., Kulashreshta P.S. \& Kadandale J.S. 2011: Characterization of TTAGG telomeric repeats, their interstitial occurrence and constitutively active telomerase in the mealybug Planococcus lilacinus (Homoptera; Coccoidea). Chromosoma 120: 165-175.

Mola L.M. \& PAPESCHI A.G. 2006: Holokinetic chromosomes at a glance. - J. Basic Appl. Genet. 17: 17-33.

Monti V., Manicardi G.C. \& Mandrioli M. 2011: Cytogenetic and molecular analysis of the holocentric chromosomes of the potato aphid Macrosiphum euphorbiae (Thomas, 1878). Comp. Cytogenet. 5: 163-172.

Nokkala S. \& NokKala C. 2004: Interaction of B chromosomes with A or B chromosomes in segregation in insects. - $\mathrm{Cy}$ togenet. Genome Res. 106: 394-397.

Nokkala S., Kuznetsova V. \& Maryańska-Nadachowska A. 2000: Achiasmate segregation of a B chromosome from the $\mathrm{X}$ chromosome in two species of psyllids (Psylloidea, Homoptera). - Genetica 108: 181-189.

NokKala S., Kuznetsova V., Maryańska-Nadachowska A. \& NoKkala C. 2004: Holocentric chromosomes in meiosis. I. Restriction of the numer of chiasmata in bivalents. - Chromosome Res. 12: 733-739.

Nokkala S., Kuznetsova V.G., Maryańska-Nadachowska A. \& Nokkala C. 2006: Holocentric chromosomes in meiosis. 
II. The modes of orientation and segregation of a bivalent. Chromosome Res. 14: 559-565.

Nokkala S. Maryańska-Nadachowska A. \& Kuznetsova V.G. 2008: First evidence of polyploidy in Psylloidea (Homoptera, Sternorrhyncha): a parthenogenetic population of Cacopsylla myrtilli (W. Wagner, 1947) from northeast Finland is apomictic and triploid. - Genetica 133: 201-205.

Nokkala C., Kuznetsova V.G. \& NokKala S. 2013: Meiosis in rare males in parthenogenetic Cacopsylla myrtilli (Wagner, 1947) (Hemiptera, Psyllidae) populations from northern Europe. - Comp. Cytogenet. 7: 241-251.

Nokkala S., Kuznetsova V.G. \& Nokkala C. 2017: Characteristics of parthenogenesis in Cacopsylla ledi (Flor, 1861) (Hemiptera, Sternorrhyncha, Psylloidea): cytological and molecular approaches. - Comp. Cytogenet. 11: 807-817.

PAPesChi A.G. \& Bressa M.J. 2006: Classical and molecular cytogenetics in Heteroptera. - Res. Adv. Entomol. 1: 1-9.

Percy D.M., Crampton-Platt A., Sveinsson S., Lemmon A.R., Moriarty Lemmon E., Ouvrard D. \& Burckhardt D. 2018: Resolving the psyllid tree of life: phylogenetic analyses of the superfamily Psylloidea (Hemiptera). - Syst. Entomol. [in press] doi: 10.1111/syen.12302

Rego A. \& Marec F. 2003: Telomeric and interstitial telomeric sequences in holokinetic chromosomes of Lepidoptera: Telomeric DNA mediates association between postpachytene bivalents in achiasmatic meiosis of females. - Chromosome Res. 11: 681-694.

Sahara K., Marec F. \& Traut W. 1999: TTAGG telomeric repeats in chromosomes of some insects and other arthropods. - Chromosome Res. 7: 449-460.

Suomalainen E. \& HalkKa O. 1963: The mode of meiosis in the Psyllina. - Chromosoma 14: 498-510.

Vítková M., Král J., Traut W., ZrzavÝ J. \& Marec F. 2005: The evolutionary origin of insect telomeric repeats, (TTAGG) $n^{*}$ Chromosome Res. 13: 145-156.

WaLton C.J. 1960: The life cycle of the hackberry gall-former, Pachypsylla celtidisgemma (Homoptera: Psyllidae). - Ann. Entomol. Soc. Am. 53: 265-277.

Received July 12, 2018; revised and accepted October 19, 2018 Published online November 5, 2018 